\title{
Leg loop Harness. Design and Method 4
}

\section{Olivier Duriez}

http://dx.doi.org/10.4314/vulnew.v78ai1.8

This design is a development of a method first used to attached VHF radio tags to small passerines (Rappole \& Tipton 1991). It has been deployed successfully on the following species: large vultures and condors (Gyps, Aegypius, Gypaetus, Vultur), small vultures (Neophron, Necrosyrtes), eagles (Aquila, Haliaeetus), kites and hawks (Milvus, Parabuteo), kestrels and falcons (Falco naumanni, F. peregrinus), species from other families (Kittiwake Rissa tridactyla, Eurasian Woodcock Scolopax rusticola, rollers Coracias garrulus, choughs Pyrrhocorax graculus, Raven Corvus corax, grouse and ptarmigan Lagopus spp.). The design was found to be unsuitable for Osprey Pandion haliaetus and pelicans Pelicanus spp.

\section{Equipment needed:}

- Teflon ribbon: $11.2 \mathrm{~mm} / 0.44$ " wide or $8.4 \mathrm{~mm} / 0.33$ " wide. Length $1.2 \mathrm{~m}$ for large vultures, $0.8 \mathrm{~m}$ for Egyptian Vultures or Golden Eagles.

- Scissors

- Clamp scissors or crocodile pliers

There are two alternative attachment methods:

1) Light non-elastic harness

- Superglue (Loctite glue)

2) Elastic harness

- Silicone tube (Versilic THT $3 \mathrm{~mm}$ diameter): https://tinyurl.com/y4l6sfas

- Cable threader or soft needle

- 2-4 earclips, double 5-7 mm: https://tinyurl.com/y2kypxn9

- A set of pliers: ringing pliers, flat pliers, pincer

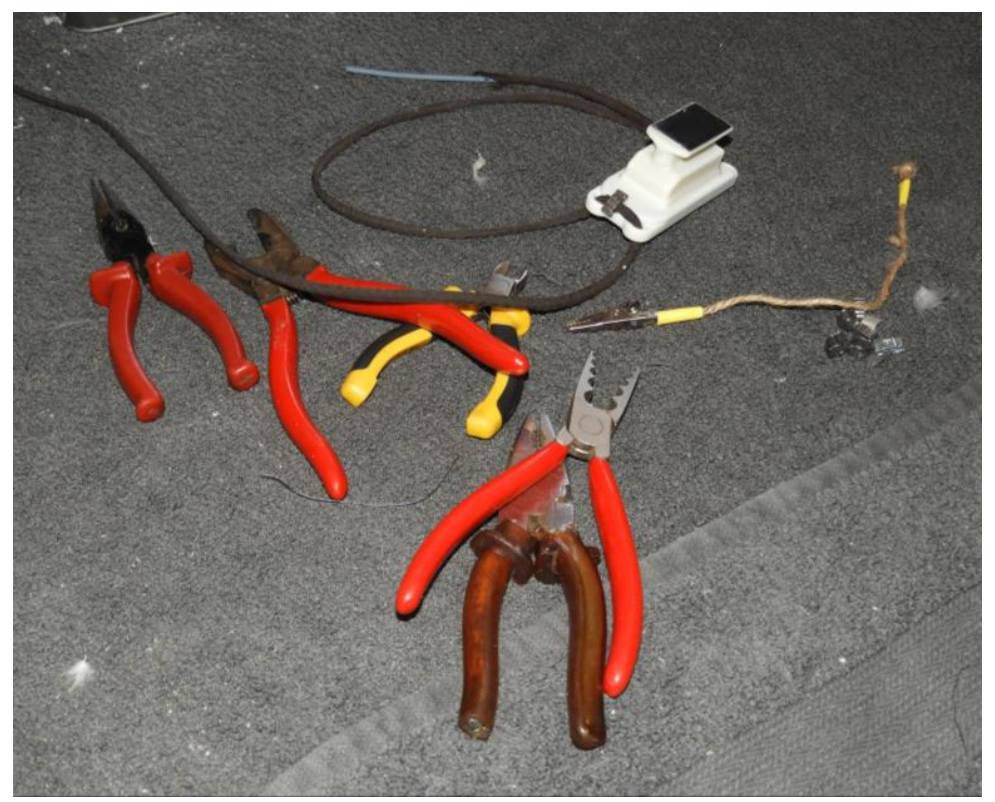

Fig. 4.1: Equipment. 


\section{Measurements and preparations: Key stages/steps}

\section{1) Non-elastic harness}

Use this option when the Teflon ribbon is narrow $(<0.8 \mathrm{~mm})$, when weight must be reduced, and when tag's lugs are narrow $(<3 \mathrm{~mm})$.

Tip: cut the Teflon ribbon diagonally instead of square at the ends (see below): this helps to insert the Teflon into the tag's lugs.

\section{2) Elastic harness}

The goal is to increase elasticity of the Teflon ribbon by inserting a silicone tube inside. Note that this increases the weight of the harness slightly and requires a large lug on the tag.

There are two options to insert the tube:

a) Use a cable threader (used for electrical wires). This only works for the large $11.2 \mathrm{~mm}$ ribbon.

b) Use a soft needle attached to the silicone tube by a thread which allows you to slowly pull the silicone inside the ribbon.
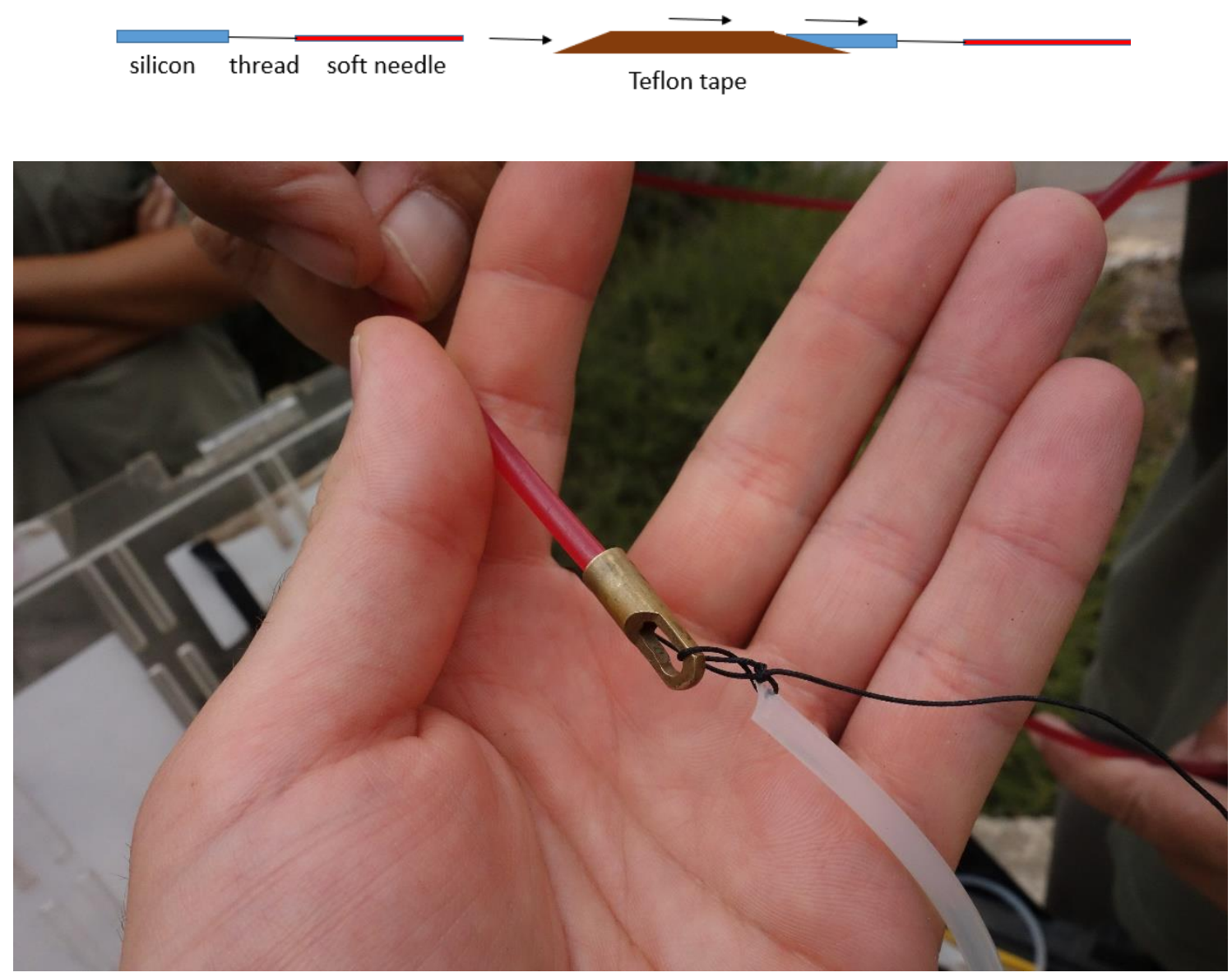

Figure 4.2: Needle and silicone tubing. 


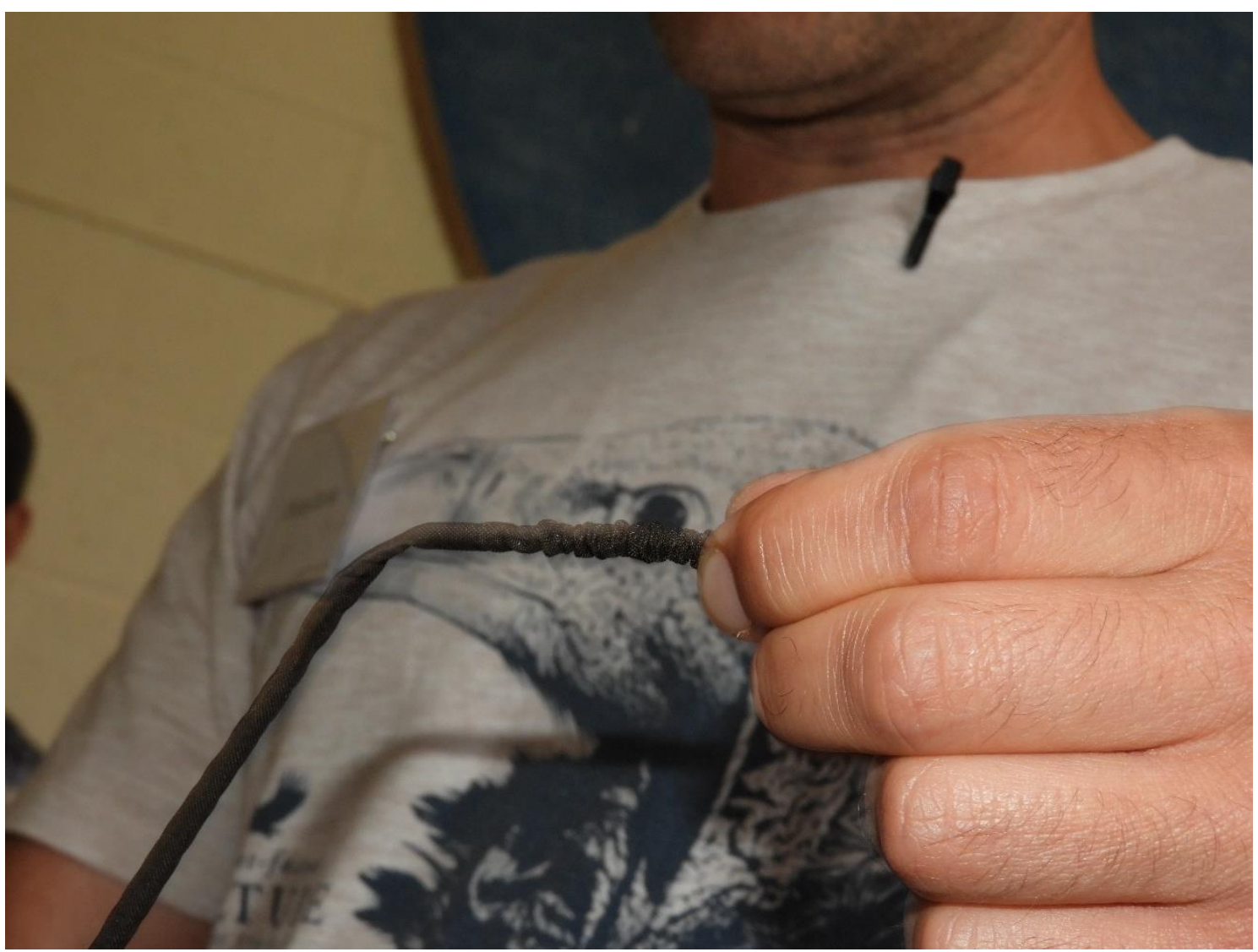

Figure 4.3: Pulling the tube through the ribbon.

Tip: cut the Teflon ribbon diagonally, instead of square at the ends: this helps to hold the Teflon while inserting the silicone tube, and also helps to insert the Teflon into the tag's lugs.

Once inserted, cut the silicone but leave $5 \mathrm{~cm}$ to stick out of the Teflon. Then pull on each side of the Teflon to create elasticity.

Before fitting the tag to the bird, it is necessary to attach the Teflon thread to part of the tag. Important: the two ribbons must have exactly the same length on each side of the tag. To do that, you can make one or two knots or attach an earclip in the middle of the thread and pass it into the tag's lugs to fix it.

If the tag has external antennas, it is easier to attach the ribbon at the rear-end of the tag (below the antennas). If the tag has no external antennas, you can attach the tag at rear or front of the tag. If your tag has one central lug, then it may be simpler to attach the ribbon at this end. 


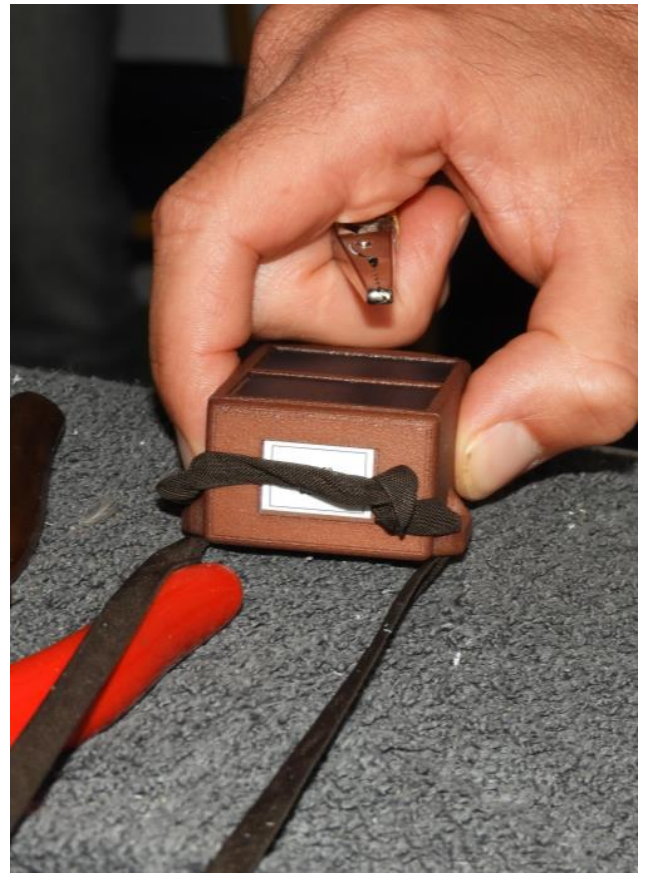

Figure 4.4: Knots on the device.

\section{Restraint and hooding}

For a large vulture, at least two people are needed, three being preferable. One person holds the headneck and the body around the wings, one person holds the legs in an extended position and spread apart and one person fits the tag

\section{Fitting to the bird}

1) Put the tag on bird's back.

2) Pass one end around the knee of one leg, and back again towards the bird's back; insert in the

3) tag lug and secure it with clamp scissors. Repeat with the second thread around the other leg.

4) Adjust the fit by pulling on both ribbons, making sure that both threads have the same length thereby ensuring that the tag will be at the centre of the back.

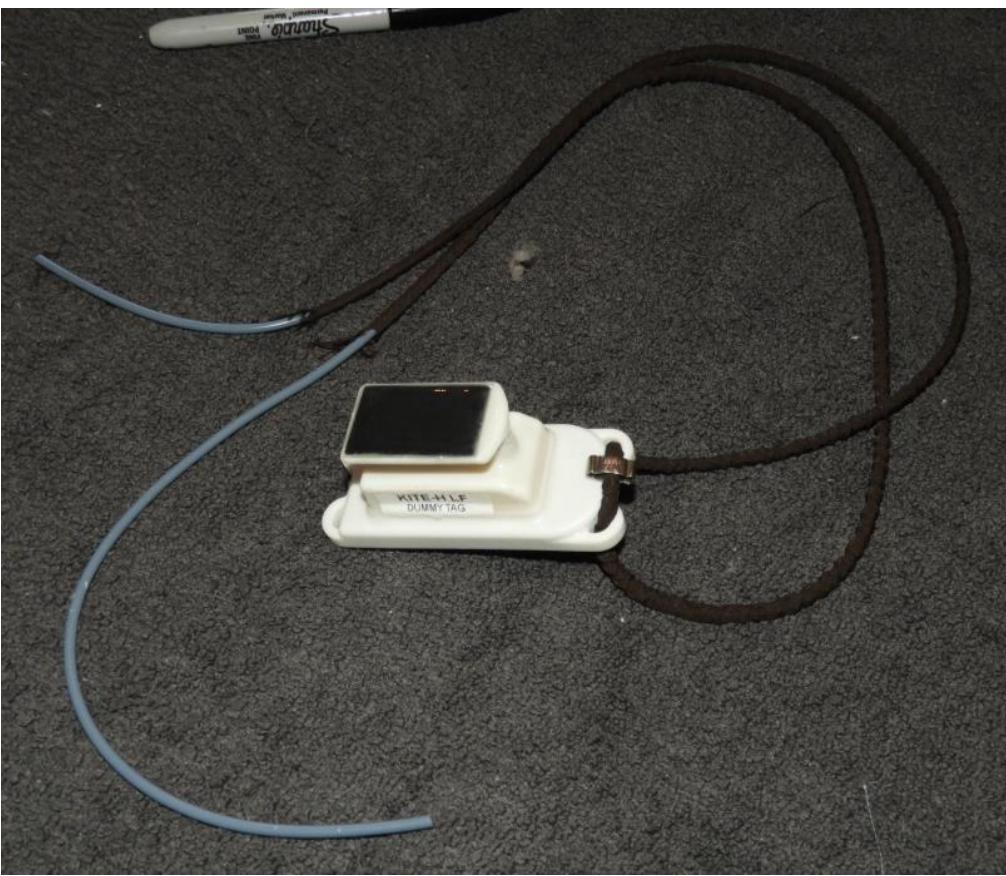

Figure 4.5: Prepared harness.

Important: check that each thread remains in the space between the knee and the body. Leave space for fingers to pass below the tag.

\section{Securing the harness: non-elastic}

5) After thread length adjustment (step 3), make a double knot with both threads;

a) put glue on the knot and make a third knot on top, to glue all knots together or

b) sew the knot with linen (flax) thread.

6) Cut the remaining parts.

7) Final check of harness fitting around the legs. This is very important. 


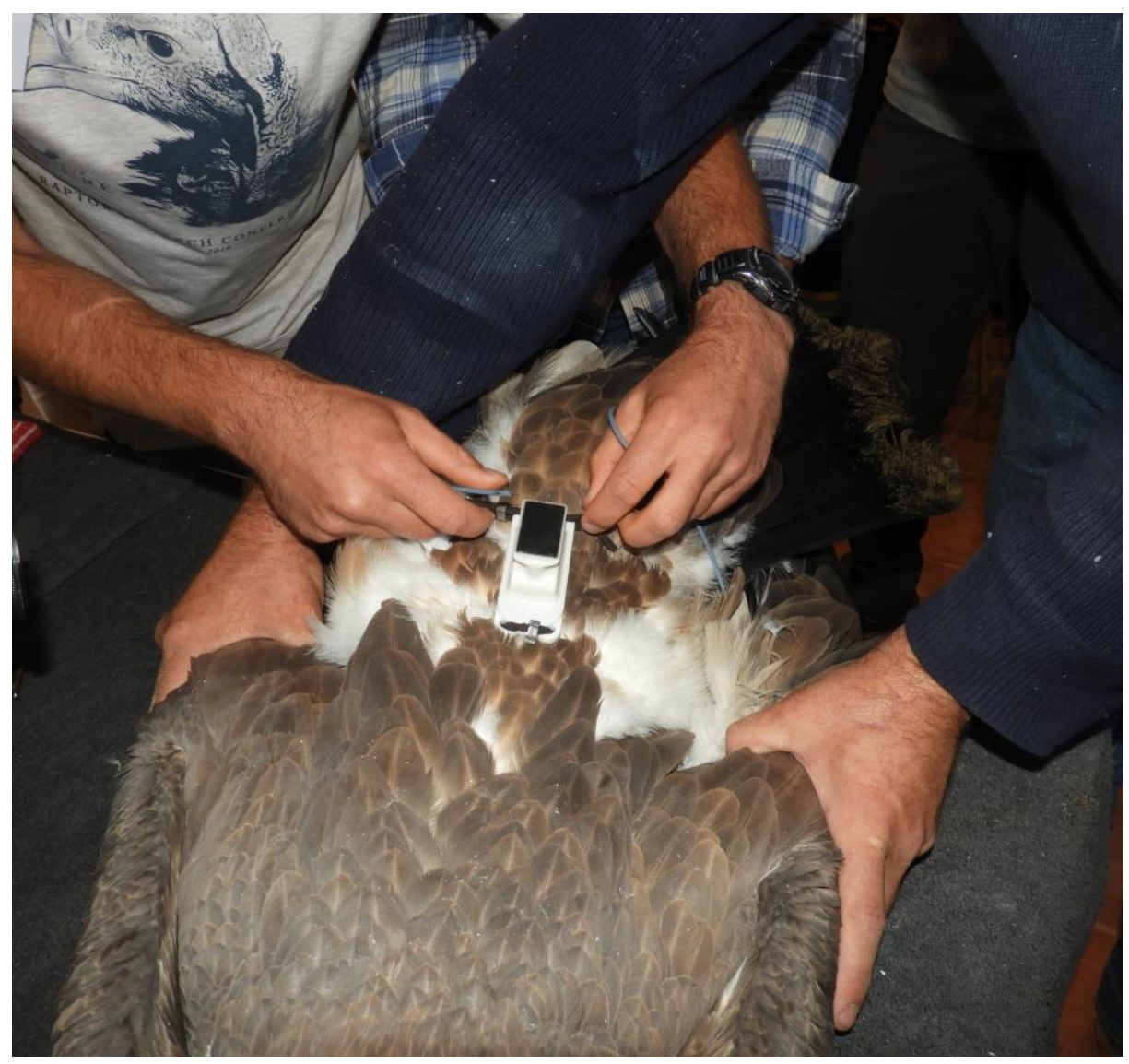

Figure 4.6: Adjusting the fit.

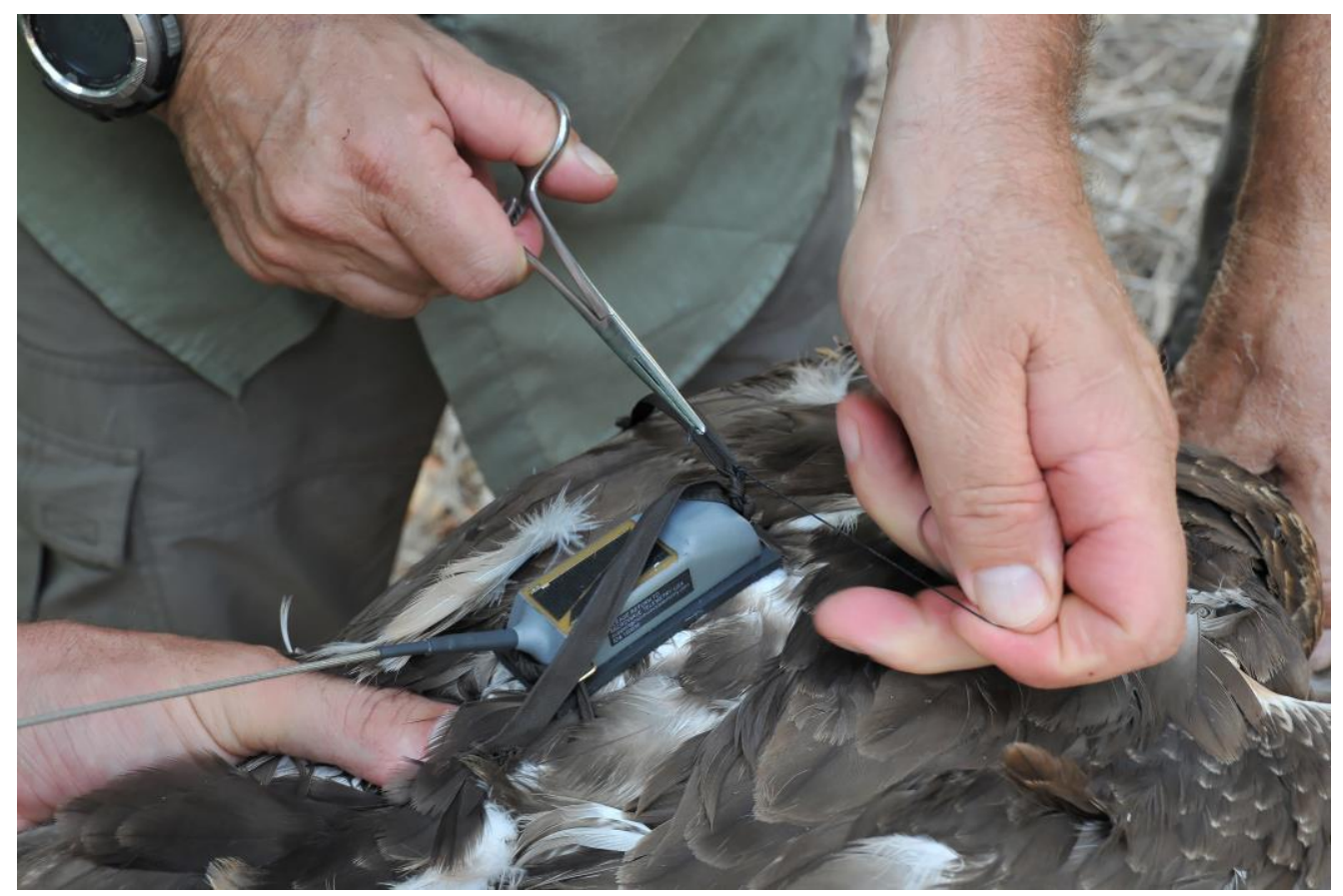

Figure 4.7 
Note that for small species, e.g. falcons, the legloops can be pre-fixed into the lugs and maintained by knots (not glued). Then after fitting around the legs (steps 2-3), the adjustment can be quicker.

\section{Securing the harness: elastic}

Before inserting the threads into the lugs (step 2), insert one or two earclips into each thread.
4) After thread length adjustment (step 3), rethread the free end of the thread into the earclips.

5) Secure the earclips with pincers. It is preferable to make the earclip rounded to prevent injuries. To do that, use two pincers to bend the earclip, then make it round with ringing pliers with holes.

6) Cut the remaining parts.

7) Final check of harness fitting around the legs. This is very important.

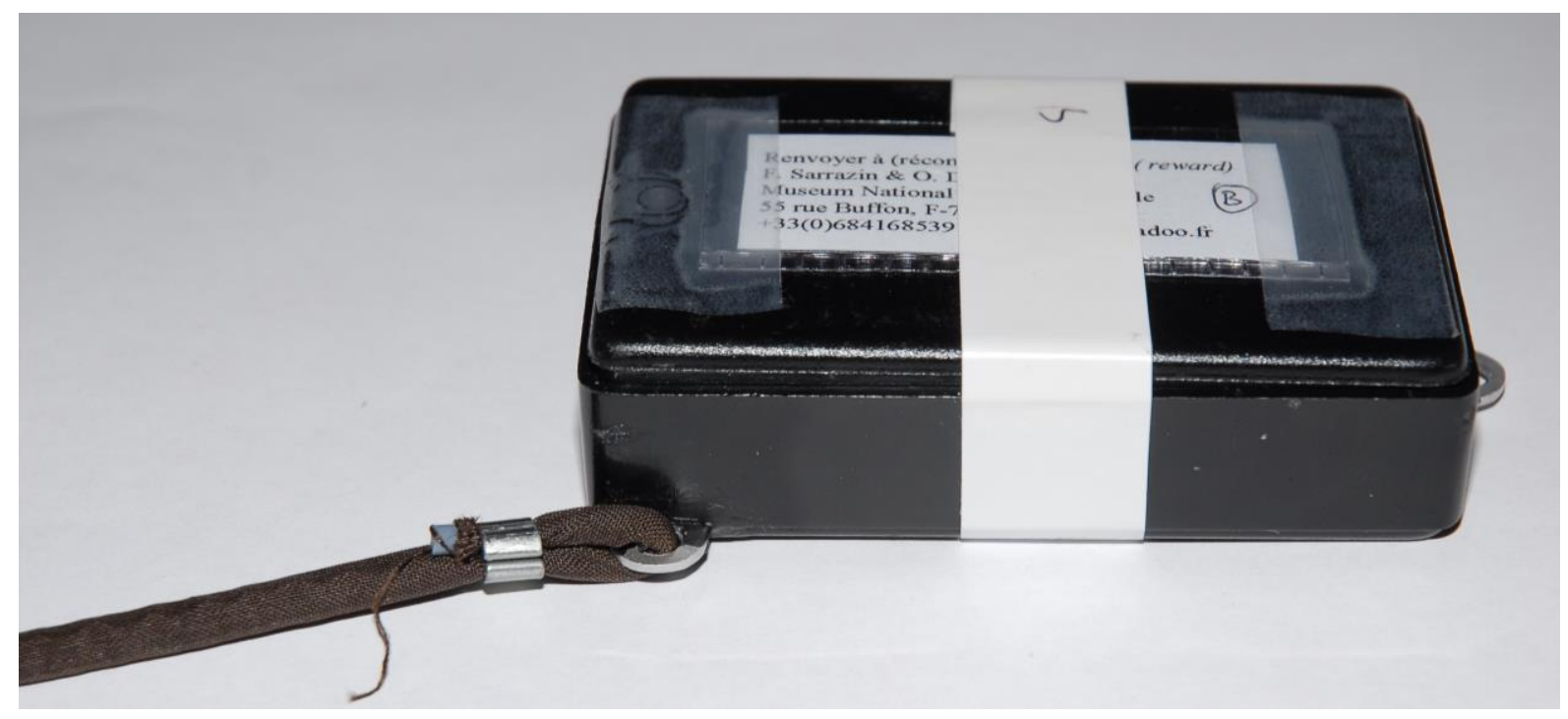

Figure 4.8: Rounded earclip.

\section{A quick harness method for doing experiments with falconry birds}

A similar leg-loop harness can be used to attach an aluminium plate, covered with Velcro, where the tag's underside is covered with the opposite Velcro. This allows multiple attach-detachments of the tags (to charge batteries and download data), without changing the harness. The harness can be adjusted to fit several birds by leaving the rear-end with an electric wire twisted around the threads. Then the harness can be quickly removed by un-twisting the electric wire. 


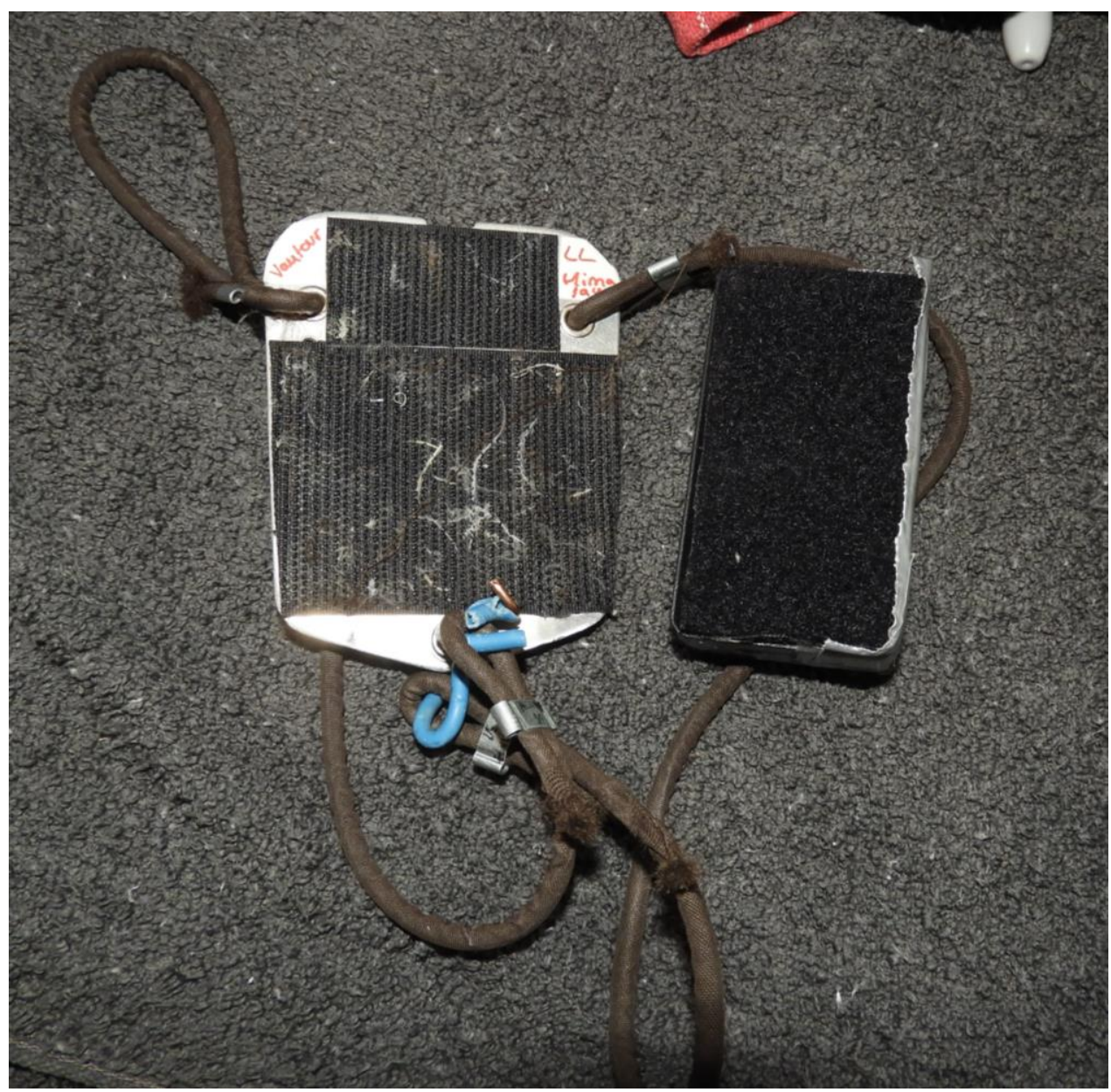

Figure 4.9: Harness with Velcro.

$* * * * * *$ 